\title{
Contraintes syntaxiques et modales des noms recteurs dans la complétive finie en français ${ }^{*}$
}

\author{
Kanté, Issa \\ Université de La Réunion, D.I.R.E. (EA 7387) \\ issa.kante@univ-reunion.fr
}

\begin{abstract}
Résumé. Cette étude propose, d'une part, une analyse typologique des complétives du nom en français, et d'autre part, elle manifeste une série de mécanismes qui sous-tendent les contraintes syntaxiques et sémantiques en jeu dans cette construction. Outre l'analyse typologique, deux contraintes sémantico-syntaxiques retiennent particulièrement notre attention : les contraintes de sélection du nom recteur (la contrainte de rection) et, dans une moindre mesure, celles portant sur la modification du nom recteur par un adjectif. Nous défendons l'hypothèse qu'outre la structure de l'énoncé et la position syntaxique du nom recteur, la contrainte de rection relève en grande partie des propriétés modales inhérentes au nom recteur. Quant à la contrainte de modification par un adjectif, nous montrons qu'elle dépend en particulier de la structure lexico-syntaxique de la complétive. Autrement dit, la fonction syntaxique qu'occupe le nom recteur dans la phrase et la relation syntagmatique dans laquelle il apparaît jouent un rôle crucial dans l'acceptabilité ou non d'un adjectif au sein du syntagme nominal recteur.
\end{abstract}

\begin{abstract}
On the one hand, this study examines the typological issues of noun complement clauses in French. On the other hand, it addresses a series of underlying mechanisms which trigger syntactic and semantic constraints in this construction, among which the head noun selectional and modification constraints. We postulate that beyond the overall structure of the sentence and the position of the head noun, the use of a noun to govern a que-clause mostly depends on the lexical modality features of that noun. As for the modification of a head noun by an adjective, in a que-clause, it depends on the syntactic function of the head noun and on the syntagmatic construction in which it appears.
\end{abstract}

\section{Introduction}

La complétive constitue un énoncé dont la complexité structurelle et catégorielle nécessitent une analyse fondée sur une distinction nette entre les différents types (complétive du nom, du verbe, de l'adjectif, etc. ${ }^{1}$. Par exemple, la complétive du nom se caractérise par ses propres formes syntaxiques et propriétés sémantiques qui doivent être identifiées et décrites en dehors du grand ensemble des complétives. Ce travail de typologie a été entrepris par certains grammairiens (Sandfeld 1965, Togeby 1985, et Wilmet 1997, entre autres), mais souvent avec des divergences de points de vue dans le choix des catégories fonctionnelles et dans la terminologie. Par exemple Wilmet $(1997: 557)$ utilise le terme « caractérisant » pour la complétive du nom - l'idée que Poil de Carotte est élégant amuse sa famille. Tandis que Togeby (1997 : 104) parle de "proposition dépendant d'un substantif » - le fait que le président du conseil fût ministre de la guerre lui semblait...). Et pendant que Wilmet (1997:556) considère comme « complétive sujet »-que je sois malade ne l'a jamais effleuré, Sandfeld (1965, p. XI-XII) quant à lui retient comme « complétive sujet »-Il est impossible que nous restions ensemble.

Cette étude poursuit deux principaux objectifs: 1) proposer une typologie fonctionnelle axée sur l'élément recteur (la catégorie grammaticale qui régit la complétive) et 2) inventorier et examiner les contraintes de sélection dont fait l'objet le nom recteur dans la complétive nominale (que nous appelons 
aussi « complétive du nom »). Dans un premier temps, nous proposons une typologie de la complétive en que visant à préciser ses grandes catégories, avec une attention particulière portée à la complétive du nom. Cette partie permettra d’isoler les catégories de complétives qui n’ont pas les mêmes propriétés intrinsèques et par conséquent méritent une analyse différenciée. La deuxième partie de l'étude a pour but d'établir un inventaire complet des différentes restrictions et contraintes syntaxiques et/ou sémantiques, même si toutes ne pourront pas faire l'objet d'un traitement approfondi ici. Néanmoins, nous examinerons de près deux niveaux de contrainte, notamment la contrainte de rection et de modification/qualification du nom recteur.

\section{Typologie des complétives}

Dans de nombreux ouvrages (Sandfeld 1965, Togeby 1985, Wilmet 1997, etc.), les auteurs proposent un classement des propositions subordonnées d'après leur fonction. Leurs analyses vont au-delà de la classification fonctionnelle de base en propositions « nominales » (les complétives, les interrogatives et les relatives indépendantes), «adjectivales » (les relatives) et « adverbiales » (les circonstancielles). Ces auteurs caractérisent les différents types de complétives (complétives du verbe, du nom, de l'adjectif, en apposition, attributs, etc.). Cette typologie dite "fonctionnelle » nous semble judicieuse dans la mesure où elle met en jeu l'interface syntaxe / sémantique. Elle nous paraît nécessaire si l'on veut expliquer les valeurs discursives des complétives et comprendre les contraintes de distributions dans chacune des catégories ainsi distinguées. Ainsi envisageons-nous ici une typologie fondée sur la relation de prédication à l'œuvre entre l'élément recteur et la complétive qu'elle gouverne. En d'autres termes, nous élaborons une typologie qui, dans un premier temps, distingue les énoncés selon la catégorie de l'élément recteur (verbe, nom, adjectif, etc.) et dans un second temps selon les critères de distribution de chaque type d'énoncé. Les complétives peuvent donc être classées en cinq² ${ }^{2}$ groupes:

i-Complétive du verbe : Marie sait que Pierre viendra. (Wilmet 1997 : 557)

ii- Complétive du nom : L’idée que Poil de Carotte est élégant amuse sa famille. (ibid)

iii- Complétive de l'adjectif : Tout heureuse que Pierre ne chante pas, Marie a promis sa venue. (ibid)

iv- Complétive de l’adverbe : Heureusement que Pierre n'a pas chanté. (ibid)

v- Complétive de la préposition : Les Paravents sont une grande pièce épique, shakespearienne, sauf que Genet donne des rôles principaux à des femmes. (U. Calgary ${ }^{3}$-Le Monde 1998)

Partant de cette classification fondée sur la catégorie grammaticale de l'élément qui régit la subordonnée, le nom recteur peut être retenu comme étant un élément caractéristique de la complétive du nom - notre objet d'étude. Ainsi, à un second niveau d'anlyse, si l'on se fonde sur la structure lexico-syntaxique au sein de laquelle le nom recteur régit la complétive, nous pouvons distinguer au moins huit types de complétives du nom, dont nous examinons ci-dessous la structure ainsi que la fonction syntaxique de la subordonnée.

\section{Typologie des complétives du nom}

\subsection{Complétive du nom sujet : [SN que]}

Dans ce type de complétive, le syntagme nominal (SN) constitue le sujet du verbe de la proposition principale $(P 1)$, ce qui impose une distribution syntaxique dans laquelle la subordonnée occupe une position médiane dans la phrase, c'est-à-dire entre le SN le fait et le SV de la proposition principale, est une nouveauté.

(1) Néanmoins, le fait que cette information soit donnée par les médias du pouvoir sans trop tergiverser est une nouveauté. (UCA-CON, Le Monde 1998) 
Au sein de cet énoncé, s’il semble clair que le SN joue la fonction grammaticale de sujet de la proposition principale $(P 1)$, il n'en va pas de même quant à sa relation vis-à-vis de la proposition subordonnée $(P 2)$. On peut sans doute dire que la complétive est une subordonnée dépendant du SN, mais qu'en est-il au juste de la relation fonctionnelle qui lie les deux constituants ? C'est la réponse à cette question qui divise les linguistes. Certains soutiennent qu'il s'agit d'une relation d'apposition et d'autres pensent plutôt que c'est un rapport de complémentation.

A la suite de Muller (2008), nous pensons que le statut de complément ou d'apposition de la complétive dépend de la structure syntaxique de l'énoncé. Muller (2008 : 402) considère que, dans un énoncé comme (1), le SN le fait entretient une relation appositive explicative avec la complétive, qui n'occupe pas une position d'argument ou de complément (obligatoire) par rapport au SN. Sur le plan syntaxique, la complétive dans (1) n'est pas obligatoire pour que la phrase soit grammaticalement correcte, d'où certainement l'idée de Grimshaw (1990) pour l'anglais, et de Muller (2002) de considérer la complétive dans une telle construction comme un modifieur ou une apposition.

(1') Néanmoins, le fait ??(que cette information soit donnée par les médias du pouvoir sans trop tergiverser) est une nouveauté. (UCA-CON, Le Monde 1998)

Cependant, sur le plan sémantique, il nous semble que la complétive dans cette phrase n’est pas facultative, puisqu'elle joue un rôle discursif nécessaire à la compréhension de l'énoncé. L'énoncé (1') avec la complétive n’a pas les mêmes propriétés de référence que sans celle-ci. De même, au niveau de la « valence » du nom recteur dans ce contexte, il nous semble que la suppression de la subordonnée dans (1') n'est pas acceptable, puisque, d'une part, le contexte ne permet pas de comprendre la référence du SN. Et d'autre part, vu que la complétive constitue l'explicitation du contenu du SN le fait, sa suppression laisserait un vide sémantique. Autrement dit, sans la subrdonnée dans l'énoncé (1') on ne peut ni saisir la référence ni le contenu sémantique du SN le fait. Pour que sa suppression soit acceptable, au moins d'un point de vue référentiel, il faudrait la présence d'éléments tels que le démonstratif ce dans (2a) ou la relative que vous avez mentionné dans (2b). Cela rendrait l'identification référentielle plus plausible, en fournissant plus de précisions sur la référence du nom - rendant ainsi plus recevable l'effacement de la complétive.

(2a) Néanmoins, ?le fait est une nouveauté. / Ce fait est une nouveauté.

(2b) Néanmoins, le fait que vous avez mentionné est une nouveauté.

En d'autres termes, la complétive dans (1') relève d'une complémentation nécessaire, non pas à cause de la valence du nom fait, mais grâce à la détermination de son contenu dans un emploi particulier. Considérant que la suppression de la complétive du nom sujet est liée au contexte, nous pouvons donc admettre que ce type de complétive est un complément contextuellement facultatif, au sens de Herbst et al. (2004) et non une simple apposition ou un adjoint. Contrairement à la complétive que nous venons d'analyser, celles des sections suivantes se caractérisent par une relation d'interdépendance sémantique et/ou syntaxique avec un constituant verbal ou prépositionnel à gauche du nom. Les éléments ou structures en question peuvent être un verbe, un présentatif ou une préposition : Vsup $+S N$ que, $V+S N$ que, Présentatif SN que, C’être SN que et Prép SN que. 


\subsection{Complétive nominale à verbe support : [Vsup SN que]}

Des verbes comme faire, prendre, donner, etc. sont appelés « verbes supports » (Vsup), lorsqu'en plus de leur emploi ordinaire, ils se combinent avec un nom, un adjectif ou un groupe prépositionnel pour construire une forme complexe, souvent fonctionnellement équivalente à un verbe (Riegel et al. 1994 : 232). Dans leur emploi avec un SN, Gross (1996: 154-155), dans une approche phraséologique, les considère comme des "verbes prédicativement vides », c’est-à-dire sans véritables arguments. Leur fonction est d'apporter à un substantif les informations de temps, de personne et de nombre. Contrairement à l'exemple (1), reproduit en (3) ci-dessous, où le SN est l'élément principal dans la rection de la complétive, on peut remarquer que le SN le risque dans (4) régit la complétive en que dans une unité sémantique avec le Vsup prendre. Se forme ainsi ce que Gross (1996) appelle « un substantif prédicatif », autour d'une relation d'interdépendance syntaxique et sémantique entre le Vsup et le SN. Dans cette relation, il nous semble que même si l'on pourrait voir dans le syntagme un SV (prendre le risque), c'est bien le SN le risque qui exprime l'essentiel du contenu sémantique de ce type de substantif prédicatif - rappelons que le verbe est prédicativement vide ${ }^{4}$.

(3) Néanmoins, le fait que cette information soit donnée par les médias du pouvoir sans trop tergiverser est une nouveauté. (UCA-CON, Le Monde 1998)

(4) Seulement je ne veux pas prendre le risque que vous puissiez prévenir quiconque à Miragoâne. (Frantext)

Muller (2008 : 402) considère la complétive régie par les structures du type avoir la certitude que P comme une construction argumentale. De même, nous dirons que prendre le risque constitue un prédicat complexe dans laquelle le verbe et le nom forment une unité sémantique dont la complétive en que est l'argument. Là réside toute la différence entre la structure Vsup + SN que P (exemple 4) et celle en $V+$ SN que $P$ (section 2.3). Le prédicat complexe prendre le risque a une structure lexico-conceptuelle faisant de la subordonnée un argument syntaxique. Là encore, les tests de l'effacement de la subordonnée (illustré dans l'énoncé 6) et de la cliticisation peuvent constituer un argument en faveur d'une telle analyse.

(5) J’ai donné l'ordre ??(que sur mes terres nul homme ne fasse obstacle à l'armée du roi...). (Frantext)

Dans cet énoncé, la complétive constitue un argument syntaxique qui complète le prédicat complexe donner l'ordre - la valence de ce prédicat exige un complément en que $P$ ou en Prép $+V$, du type donner qu'il vienne ou l'ordre de venir. En outre, sur le plan sémantique, sans la subordonnée, on ne peut pas identifier le contenu de l'ordre en question. Nous pouvons donc postuler que l'effacement de la complétive après Vsup + SN (recteur) est a priori problématique, sauf si la valence de Vsup + SN permet la non-réalisation du complément selon le contexte. C'est l'hypothèse défendue par Godard (1996 : 305) lorsqu'elle affirme ceci : « on admettra donc que la complétive est argument d'un prédicat complexe dans les structures à V support, alors que ce n'est pas un argument dans le seul GN [complétive régie par un nom sujet]. C'est dans cette mesure seulement qu'elle peut donner lieu à la cliticisation [par en] ».

L’idée de Godard (1996 : 304) est que si la complétive du nom est argument, elle doit être cliticisable par en, même si l'acceptabilité d'une telle transformation dépend en général du verbe lexical qui sert de support au nom. Les énoncés (4 et 5) repris respectivement en $(6 \mathrm{a} / \mathrm{b})$ se soumettent à la règle de cliticisation par en.

(6a) Que vous puissiez prévenir quiconque à Miragoâne, je ne veux pas en prendre le risque.

(6b) Que sur mes terres nul homme ne fasse obstacle à l'armée du roi, j’en ai donné l'ordre.

Outre l'effacement de la complétive (6b), la possibilité de cliticisation avec la dislocation à gauche de la proposition en que de ces énoncés français conforte l'hypothèse selon laquelle la complétive à verbe support est bien un complément obligatoire, donc un argument. Autrement dit, vu que prendre le risque que $P$ et donner l'ordre que $P$ sont cliticisables par en et que la suppression du complément phrastique 
rend l'énoncé peu acceptable, nous pourrions y voir un statut d’argument (complément syntaxique) de la subordonnée en que.

\subsection{Complétive du nom objet [V + SN + que]}

Dans l'énoncé ci-dessous, de même que dans la structure à verbe support supra, l'apparition d'un verbe à gauche du SN recteur ouvre une place de sujet occupée par un élément autre que le nom recteur, les enquêteurs. Cette distribution confère généralement à la complétive en que une position en fin d'énoncé. Il y a une interdépendance syntaxique entre le verbe écarter et le SN l'hypothèse pour régir la complétive.

(7) Les enquêteurs n'écartent pas l'hypothèse qu'un film vidéo de la collision aérienne ait pu être pris par l'un des nombreux curieux venus admirer le Norway. (UCA-CON, Le Monde 1998)

Dans ce type de construction, la subordonnée en que joue la fonction de complément dont l'effacement est conditionné par l'existence d'un élément contextuel aidant à identifier le référent du SN. L'énoncé (7) sans la subordonnée peut être syntaxiquement acceptable, mais pas en termes de statut informationnel, ce qui implique que la complétive dans cet énoncé n’est pas un adjoint apposé.

(7’) ?Les enquêteurs n’écartent pas l’hypothèse.

Le verbe écarter a son complément l'hypothèse, mais ce dernier sans la complétive (7') est donc discursivement incomplet. Ce SN nécessite la présence d'un autre élément avec lequel il entretient une relation d'explicitation, telle que que $P$ dans (l'exemple 7). C'est justement cette relation qui rend la présence de la complétive plus ou moins obligatoire selon l'environnement syntaxique ou le contexte discursif. Par exemple, la simple introduction d'un autre déterminant comme votre, ayant une fonction référentielle plus marquée dans les enquêteurs n'écartent pas votre hypothèse, rendrait l'énoncé sans la subordonnée davantage acceptable.

Partant de ces analyses, nous pouvons conclure que la complétive en que régie par un SN en position objet est un complément syntaxiquement facultatif. On a vu dans (7’) que sa suppression pose problème d'un point de vue sémantique, mais pas syntaxique, dans la mesure où elle n'entraîne pas nécessairement une inacceptabilité syntaxique. Fonctionnellement, cette construction se distingue ainsi de la complétive à verbe support dont la subordonnée est un argument syntaxique (complément obligatoire).

Outre les cas de dépendances sémantiques et/ou syntaxiques entre le $\mathrm{SN}$ et le verbe, [Vsup $+S N$ que], $[V$ $+S N$ que], il existe d'autres constructions dans lesquelles le SN dépend syntaxiquement des structures présentatives-existentielles (il y $a+S N$ que) ou des extrapositions (c'être + SN que).

\subsection{Complétive nominale à structure présentative : [il y avoir/voilà/voici + SN + que]}

Les présentatifs, qui sont des expressions (non)actualisables, constituent une sorte de syntagme verbal permettant l'introduction d'un SN recteur, avec lequel il entretient une relation d'interdépendance syntaxique pour régir la complétive. Notons que dans cette relation c'est bien le SN qui constitue l'élément central dans la rection : l'espoir que $P$ et non pas le présentatif *il y a que $P$ ou *voici que $P$.

(8a) Il ajoute : comme il y a peu d'espoir que l'Irak change d'attitude, la charge de la preuve revient aux pays qui estiment qu'il y a un autre choix que la force. (UCA-CON, Le Monde 1998)

(8b) Le diaporama peut être visionné sur les ordinateurs personnels ou en circuit fermé. Voilà la preuve que, même avec des moyens modestes, on peut ouvrir toute grande la porte à la francisation.

(http://www.olf.gouv.qc.ca/ressources/place_publique/francofete/francofete_2004/laureats_ftc_20 04.html, consulté en juin 2015) 
(8c) Pour Martin-Chauffier, voici une preuve que son journal n'est plus celui que Roger Thérond avait conduit haut et fort. (Le nouvel Economiste - n²1386 - Du 3 au 9 mai 2007, consulté en juin 2015)

L’analyse des énoncés (8a/b/c) en constituants immédiats - Présentatif + SN + P2 : ((Présentatif) - (SN2) - (P2)) montre que le complément du présentatif (SN2) est certes l'élément qui joue le rôle principal de recteur de la complétive $P 2$, mais il ne remplit pas seul cette fonction. La relation entre les constituants montre en effet que c'est avec toute la structure présentative que le nom régit la complétive. Dans ces énoncés, le syntagme de rection présentatif + SN joue un rôle comparable à celui de Vsup + SN.

Les présentatifs, comme l'indiquent Arrivé et al. (1986: 565), constituent à eux seuls des groupes verbaux qui introduisent des noms, des infinitifs ou des propositions en que. Dans le même ordre d'idée, Chevalier et Léard (1996 : 56) incluent il y a dans la liste des verbes supports de la même manière que nous considérons prendre (voir énoncé 4). Comme le font ces verbes, les présentatifs formeraient avec le nom une sorte de prédicat complexe dans la rection de la complétive. Ces observations doivent-elles nous conduire à considérer que la complétive régie par présentatif + $S N$ est un complément argumental ? Cela semble être le cas si l'on se fie au seul test de cliticisation par en :

(9a) ?? Que l'Irak change d'attitude, il y (en) a peu d'espoir.

(9b) Que, même avec des moyens modestes, on puisse ouvrir toute grande la porte à la francisation, en voilà une/la preuve.

(9c) Que son journal ne soit plus celui que Roger Thérond avait conduit haut et fort, en ?voici une/la preuve.

Mais cette question est beaucoup plus complexe qu'il n’y paraît. Il faut souligner que le parallélisme entre $[V s u p+S N$ que $]$ et [Présentatif $+S N$ que] ne tient pas à tous les niveaux. Par exemple, le premier forme une unité sémantique et syntaxique beaucoup plus forte que le second. Dans la complétive à verbe support, le prédicat complexe correspond à un même sens (prendre le risque que $P=$ risquer $P$ / avoir la certitude que $P=$ être sûr que $P$ ) alors que les présentatifs (il y a peu d'espoir / voilà la preuve) ne sont porteurs d'aucun autre sens que celui qu'ils ont dans la plupart de leurs emplois de base.

Sur le plan syntaxique, le SN joue la fonction d’objet vis-à-vis du présentatif. Les noms (danger, espoir et preuve) peuvent tous apparaître dans $[S N+$ être +que] et la suppression de la complétive n'entraînerait pas nécessairement une inacceptabilité syntaxique. Mais là encore, comme dans la complétive du nom sujet, la suppression de que $P$ peut entraîner une incomplétude discursive. L’énoncé voilà la preuve sans la subordonnée manquerait de contenu discursif, puisqu'il ne suffit pas pour savoir à quoi renvoie le SN la preuve. A cet égard, nous pouvons indiquer qu'excepté la cliticisation, les autres tests (la possibilité d'effacer le complément phrastique et l'insertion de la copule) montrent plutôt que la complétive du nom d'un présentatif est un complément facultatif. Par conséquent, nous devons admettre que les similitudes entre la complétive nominale régie par un présentatif et celle régie par Vsup $+S N$ ne sont que partielles. Elles existent à quelques niveaux syntaxiques, parmi lesquels le caractère de support syntaxique du présentatif et la position de la complétive dans la structure syntagmatique. La complétive est un complément obligatoire (ayant un rôle d'argument syntaxique et apportant le contenu sémantique) dans l'un, [Vsup + SN que] et un complément facultatif, du moins syntaxiquement, dans l'autre, [Présentatif + SN que].

L’intégration du nom recteur et de la complétive dans les structures que nous venons d'étudier, en l'occurrence il y $a+S N$ que $P$, reflète une distribution syntaxique très proche, en surface, de la complétive dite "extraposée » : ce + être + SN que. La tentation d'assigner un statut de support à P1 est assez forte dans la complétive du nom extraposée. Dans ce type de complétive, le nom recteur est introduit par le pronom neutre ce et l'auxiliaire être. Dans la section ci-dessous, nous traitons de ce type de complétive, et nous montrons qu'il a plus de traits communs, notamment sémantiques et pragmatiques, avec la complétive sujet qu'avec celle régie par le présentatif (il y a $+S N$ que $P$ ). 


\subsection{Complétive nominale extraposée : [c'être SN que]}

Par complétive nominale extraposée $e^{5}$, nous désignons la construction dans laquelle la position sujet de $P 1$ est occupée par un pronom impersonnel, le plus souvent (ce) en français: [que $P+V+\hat{e} t r e+S N=>c e+$ $V+$ être + SN que P].

(10a) Tant que vous verrez quelqu'un dans l'antichambre des magistrats et des tribunaux, le gouvernement ne vaut rien. C'est une horreur qu'on soit obligé de demander justice. (SAINT-JUST (1793), Fragments sur les institutions républicaines. Ed. 2003 : 162)

(10b) Qu’on soit obligé de demander justice (cela) est une horreur.

Les énoncés $(10 \mathrm{a} / \mathrm{b})$ illustrent les cas d'extrapositions où la complétive, qui est censée être le sujet logique, laisse sa place au pronom neutre ce. La complétive extraposée (comme dans 10a) est une variante de la complétive antéposée (10b), qui est, elle aussi, à rapprocher de la complétive régie par un SN sujet :

(11) Le fait/l'idée qu’on soit obligé de demander justice est une horreur.

Outre les cinq types de complétives que nous venons d'analyser, nous énumérerons brièvement cidessous les trois autres types de complétives, même si faute d'espace, nous n'en ferons pas une présentation détaillée (cf. Kanté 2013 et 2011).

\subsection{Complétive nominale prépositionnelle : [Prép SN que]}

Comme les présentatifs voici, il y a, etc. (section 2.4), certaines prépositions peuvent servir de support à un SN pour régir une complétive. Dans certains cas (sous prétexte que ou à telle enseigne que), la présence de la préposition est même indispensable dans la rection de la complétive. D'un point de vue sémantique, on peut distinguer deux sous-types dans lesquels la préposition sert d'élément support au nom recteur.

\section{a) Les locutions prépositives explicatives-causales :}

(12) Mais en outre elle m’avait emprunté la somme nécessaire à l'achat d’une voiture, sous prétexte que les traites constituent une véritable escroquerie. (Frantext)

\section{b) Les locutions prépositives additives-exceptives:}

(13) A priori, les deux affaires n’ont pas de rapport hormis le fait que le général Rondot a été missionné dans les deux cas. (http://www.politique.net/2007122702-le-compte-secret-dejacques-chirac-au-japon.htm, consulté en juin 2015)

Notons que les locutions sous prétexte que et hormis le fait que, et bien d'autres, sont souvent perçues comme des formes figées non analysables syntaxiquement. Cependant, comme le souligne Gross (1996 : 127-137), le traitement par les grammaires scolaires des «locutions conjonctives » telles que au fur et à mesure que, sous prétexte que, à telle enseigne que, etc. occulte un fonctionnement linguistique très complexe. Elles ne sont pas toutes figées et la structure interne de la plupart d'entre elles peut subir des variations analysables syntaxiquement ${ }^{6}$.

\subsection{Complétive attribut du nom sujet : [SN être que]}

La complétive attribut constitue un des types dans lesquels la subordonnée en que est de fait nonadjacente au nom recteur : 
(14) L’éminent membre du VVD Gerrit Zalm, qui a personnellement 'soufflé' Hirsi Ali au PvdA, ne voit pas de raison lui non plus de tenir un nouveau débat parlementaire. L'image est que les Pays-Bas ne veulent pas payer pour la sécurité d'Hirsi Ali. C’est tout à fait injustifié, nous payons ici. Les autorités américaines ne le font pas quand elle est là-bas. (http://www.ambafrance-nl.org/france_paysbas/spip.php?article9287, consulté en juin 2015)

\subsection{Complétive du nom « discontinue » : [SN + V que $]$}

Le mode de construction de cette complétive consiste à placer le verbe de $P 1$ entre le nom recteur et la complétive qui, sinon, sont normalement adjacents : $[S N$ sujet + que $P+V=>S N+V+q u e P]-$ ce processus est aussi qualifié de disjonction.

(15a) AJIEP (Sud-Soudan) de notre envoyée spéciale la rumeur a couru qu’une distribution de maïs allait avoir lieu vendredi matin. (UCA-CON, Le Monde 1998)

(15b) La rumeur (qu'une distribution de maïs allait avoir lieu vendredi matin) a couru.

A l'issu de l'analyse des énoncés dans cette section (exemples 1 à 15), nous pouvons constater que l'élément caractéristique commun reste le nom recteur qui joue un rôle syntaxique et sémantique prépondérant dans la construction de la complétive. En revanche, nous constatons également que les différences de structure sous-tendent des différences de fonction de la subordonnée par rapport à ce même nom recteur. Bien que ce dernier soit commun aux huit types, leur organisation interne reflète différents mécanismes syntaxiques. Dans l'exemple (1), la complétive dépend directement du SN le fait auquel elle est liée par " prédication simple » (dans le sens d'un seul prédicat nominal). Alors que la complétive dans les exemples (4-15), certes, dépend du SN recteur, mais contrairement à (1), la relation s'établit grâce à ce qu'on pourrait appeler une «prédication complexe». En effet, pour régir la complétive, le SN s'associe à un verbe support (exemple 4), dépend d'une structure présentative-existentielle (c'est une horreur que...) ou encore apparaît dans une structure attributive (le problème est que...). Outre ces structures, le SN recteur peut aussi avoir comme support une préposition pour régir une complétive (12 et 13), où la préposition joue un rôle prépondérant dans la rection.

Une telle typologie permet d'établir d'une part la distinction entre les différents types de complétives, et d'autre part de proposer une classification selon les propriétés distributionnelles du nom recteur au sein de la complétive. Une telle distinction en amont permet également de mieux poser la question des contraintes distributionnelles que nous abordons ci-dessous.

\section{Les contraintes distributionnelles dans la complétive du nom}

La construction de la complétive est soumise à de multiples contraintes aussi bien sur l'axe paradigmatique que syntagmatique, contraintes qui se manifestent au niveau du mode, de l'adjacence, de la rection, détermination, et modification. Notons que la complétive, qu'elle soit verbale, nominale, adjectivale ou adverbiale, est soumise aux trois premières contraintes (mode, adjacence et rection). Ces contraintes apparaissent à différents niveaux et peuvent, de surcroît, prendre plusieurs formes en fonction des éléments de chaque niveau. Elles ont parfois déjà été repérées dans la littérature ${ }^{7}$ mais, à notre connaissance, elles n’ont pas été relevées de façon oppositive et exhaustive. Les sections suivantes illustrent ces différents niveaux/types de contrainte, tandis que la section 4 propose une étude un plus détaillée de la contrainte de rection et de modification du nom recteur.

\subsection{La contrainte de détermination}

Dans la complétive nominale, la détermination du nom présente toute une gamme de contraintes. Outre ce qu’illustrent les énoncés (16a/b), d’autres contraintes existent, parmi lesquelles la possibilité d'employer 
les possessifs ou les démonstratifs avec certains noms (son/cette idée que Poil de Carotte est élégant amuse sa famille) et l'agrammaticalité des possessifs avec des noms comme (*son/ton fait que P).

(16a) L’idée que Poil de Carotte est élégant amuse sa famille.

(16b) *Une idée que Poil de Carotte est élégant amuse sa famille.

En plus des structures de prédication simple [SN + que], les possessifs et les démonstratifs peuvent apparaître avec certains noms (détail, difficulté, particularité) lorsqu'ils sont dans une structure de prédication complexe $[S N+$ Vsup. + que $]$ : il a cette particularité qu'il est gaucher. On l'a reconnu à ce détail caractéristique qu’il portait un chapeau gris, Le Goffic (1993 : 540)

\subsection{La contrainte de mode}

Les énoncés (17a/b et 18a/b) montrent deux fonctionnements des noms recteurs dans la sélection des modes.

(17a) Paul a l’impression que Médor est ici. (Gross 1975 : 53)

(17b) Paul a l’impression que Médor *soit ici. (ibid)

(17c) Paul n’a pas l'impression que Médor soit ici. (ibid)

(18a) Madame de Rénal eut la folle idée que cet avertissement n'était qu'un prétexte pour ne pas la voir. (U. Virginia-Etext)

(18b) Je n’aime pas l’idée qu'elle soit hospitalisée là-bas. (Togeby 1985 : 96)

Dans les grammaires, on note souvent qu'il existe d'un côté des noms comme impression ou bruit qui n'accepteraient que l'indicatif ${ }^{8}$, et de l'autre des noms comme idée qui se soumettent à l'alternance indicatif / subjonctif. L'analyse d'énoncés extraits de corpus semble indiquer que le problème est plus complexe qu'il ne paraît. En effet, des auteurs comme Martin (1983), Kupferman (1996) et Korzen (2003) adoptent une analyse fondée sur le principe que la question du mode après les noms recteurs ne doit pas être abordée en termes stricts de "tel nom exige tel mode »; mais plutôt en termes de tel nom privilégie tel ou tel mode. Cette approche nous semble en effet judicieuse puisqu'elle permet de rendre compte de nombreux cas où le subjonctif ou l'indicatif apparaissent dans des structures où on ne les attendait pas. Dans Kanté (2010 et 2011), nous défendons l’hypothèse qu'une typologie des noms recteurs en classes modales (modalité lexicale) pourrait aider à mieux comprendre cette problématique. Une telle méthode peut être un outil pertinent permettant non seulement de dégager les tendances qui sous-tendent l'alternance indicatif / subjonctif, mais surtout d'expliquer pourquoi telle classe modale de nom ou tel nom au sein d'une classe privilégie tel mode.

Quant aux noms ou verbes qui se soumettent systématiquement à l'alternance indicatif / subjonctif, Soutet (2000 : 61-66) explique que cette « double commande modale » est due à deux mécanismes sémantiques: pour le verbe, c'est la polysémie, et pour le nom recteur c'est plutôt un phénomène d'implication sousjacente qui serait à l'origine de cette alternance. Selon Soutet, les verbes comme admettre ou comprendre acceptent l'indicatif et le subjonctif parce qu'ils ont deux signifiés distincts. Admettre par exemple régit l'indicatif lorsqu'il signifie " être d'accord sur le fait » (Pierre admet que Paul est parti) et le subjonctif lorsqu'il signifie «trouver une justification à » (Pierre admet que Paul soit parti). Quant au nom recteur, c'est de par les implications de sa valeur de jugement qu'il permet l'emploi des deux modes. Quand il renvoie à un monde factuel, où il pose la réalité du contenu propositionnel, il régit l’indicatif. Et quand il évoque un monde contrefactuel, il commande le subjonctif. Ahern (2005), dont l'étude porte sur la double commande modale en espagnol, semble défendre une position similaire lorsqu'elle affirme que l'alternance indicatif / subjonctif est due à l'intention d'assertion ou de non-assertion du locuteur. Cette position rejoint l'analyse de Celle (2006 : 74-91), lorsqu'elle considère le subjonctif comme un marqueur de « non-prise en charge » en français et en allemand. Elle indique que le subjonctif marque « [l'] absence 
de prise en charge de la part de l'énonciateur et [l'] absence de tout commentaire énonciatif concernant la validité de la relation prédicative ${ }^{9}{ }^{\prime}$.

Notons enfin que l'approche de Soutet et celle d'Ahern pourraient renforcer l'hypothèse que le nom recteur serait un marqueur lexical de modalité (voir section 4 ci-dessous et Kanté 2010). Ces deux auteurs reconnaissent que, (1) c'est le nom recteur qui commande le choix du mode (relation modale entre le sémantisme du nom et le sens du mode), et que (2) le mode est plutôt un marqueur de modalité d'orientation. De ce fait, si le rôle du mode est de marquer l'orientation énonciative, le type de modalité serait donc marqué par d'autres éléments de l'énoncé, parmi lesquels le nom recteur. En d'autre termes, si le mode détermine l'intention d'assertion ou de non-assertion du locuteur, le SN régissant la complétive indiquerait si la modalité est épistémique (l'impression / la certitude que $P$ ), déontique (la recommandation / l'exigence que $P$ ), volitive (la volonté / le souhait que $P$ ), etc.

\subsection{La contrainte d'adjacence}

Dans la plupart des énoncés que nous avons vus jusque-là, on peut noter que la complétive du nom apparaît en fin de phrase (position la plus courante), mais elle peut aussi s'insérer entre le nom recteur et le SV de la proposition principale, comme dans l'exemple suivant.

(19a) Le fait qu'il soit venu m’inquiète. (Muller 1996 : 32)

(19b) *Le fait m’inquiète qu'il soit venu.

Là encore, le type de structure lexico-syntaxique (présence ou non d'éléments supports auprès du nom recteur) semble être déterminant dans le choix de la position de la complétive dans la phrase. La complétive serait insérée entre le nom recteur et son SV, lorsque celui-ci se trouve dans une structure lexico-syntaxique simple entre le nom recteur et la subordonnée ( $S N+$ que : l'idée que Poil de Carotte est élégant amuse sa famille). Elle serait en fin de phrase lorsque le nom recteur apparaît dans une structure lexico-syntaxique complexe. L’énoncé (19b) illustre la contrainte structurelle qu'engendre la mise en position finale de la complétive d’un prédicat nominal simple. Notons que notre analyse ne tient compte que des complétives dont le verbe de la proposition introductive est à la forme finie, les complétives introduites par des formes non finies (partant du fait que... / en se basant sur le fait que...) en sont donc exclues. Ces quelques cas illustrent la diversité et la complexité des contraintes et restrictions dans la complétive du nom. A défaut de les étudier toutes en détail ici, nous examinons cidessous deux cas.

\section{Les contraintes de rection et de modification}

\subsection{La contrainte de rection}

Les énoncés ci-dessous illustrent la contrainte de sélection qui pèse sur la classe des noms susceptibles de régir une complétive. Le nom idée peut régir la complétive avec ou sans l'aide d'un verbe (support), contrairement au nom esprit.

(20a) L’idée que Poil de Carotte est élégant amuse sa famille. (Wilmet 1997 :557)

(20b) *L'esprit que Poil de Carotte est élégant amuse sa famille.

(20c) Il défend *l'esprit / l’idée que Poil de Carotte est élégant.

L'objectif de cette section est d'illustrer les traits sémantiques permettant aux noms recteurs tels que conviction, certitude, fait, idée, rumeur, vérité, philosophie, besoin, souhait, vœu, chose, etc. de régir une complétive en que. Leclère (1971) réalise une étude étendue sur les contraintes dans la complétive, mais sans séparation entre la forme finie de la complétive du nom et la complétive infinitive qu’il considère d'ailleurs comme la forme réduite de la première. Cette étude sur les contraintes morphosyntaxiques a le 
mérite d'être l'une des plus détaillées, mais elle ne distingue pas entre les propriétés qui sont valables pour la complétive infinitive et celles qui ne le sont que pour la forme finie de la complétive du nom. Par exemple, Leclère (1971) aborde à la fois un "substantif opérateur » comme impression, qui peut être suivi aussi bien d'une complétive finie que d'un infinitif (Luc a l'impression qu'il a bien travaillé. / Luc a l'impression d'avoir bien travaillé. Leclère $1971: 61$ ), et un autre comme bêtise, qui ne peut être seulement suivi de l'infinitif : Ève a eu la bêtise de refuser mon aide. (Leclère $1971: 61$ ) / *Ève a eu la bêtise qu'elle a refusé mon aide.

Ainsi, une telle étude ne permet pas de comprendre sur quoi repose cette contrainte de sélection. Autrement dit, en étudiant les complétives sans séparation stricte entre les différentes catégories (complétive finie et complétive infinitive ou complétive du verbe et du nom), les conclusions risquent d'être confrontées à plus de contre-exemples venant d'une catégorie ou de l'autre. En outre, en traitant à la fois la complétive finie et infinitive, on neutralise de facto la question de l'alternance modale - la contrainte de mode se manifestant uniquement dans la première catégorie.

Il est aisé de constater à quel point la contrainte de rection est très forte dans la sélection d'un nom pour construire une complétive : Luc a l'impression/*le sourire] qu'il a bien travaillé. Il reste à démontrer comment la sélection du nom s’opère dans la construction de la complétive du nom et pourquoi des noms comme fait, impression, philosophie peuvent régir la complétive, tandis que d'autres comme sourire, inflation, linguistique ne le peuvent pas dès lors que la proposition en que est une complétive ? Comment et sous quelles conditions cette contrainte se manifeste-t-elle ? Y aurait-il des traits intrinsèques à certains noms qui font qu'ils peuvent (ou ne peuvent pas) régir une complétive du nom ? Dans la littérature, un consensus semble se dégager affirmant que les propriétés sémantiques des prédicats sont déterminantes dans la rection des complétives (cf. Boone 1996, Chevalier et Léard 1996, ou Khalifa 2004 et Perkins 1983 pour l'anglais). Il faut toutefois noter que cette observation reste souvent vague, puisqu'elle ne dit pas quelles sont les propriétés sémantiques que doit posséder un nom afin qu’il puisse régir une complétive.

Une première perspective de travail consiste à se demander si une des propriétés en question ne serait pas la valeur de modalité lexicale des noms recteurs. Notre hypothèse est la suivante : la modalité serait un des traits centraux dans la sélection des noms recteurs. A cet égard, la contrainte de sélection du nom recteur serait, au moins en partie, liée à la modalité. Kanté (2011 et 2010) propose une analyse et une classification des noms recteurs selon la valeur de modalité lexicale qu'ils peuvent exprimer. L’idée sousjacente est la suivante : pour qu'un nom puisse régir une complétive, il doit avoir une valeur [+MODALITE] intrinsèque et l'inexistence [-MODALITE] de cette valeur-là pourrait être une source de blocage de la rection, comme l'illustre le contraste entre les noms croyance vs religion ou philosophie vs linguistique ${ }^{10}$ dans l'exemple suivant : La forme du songe choisie par l'auteur italien illustre cette croyance [*religion/ philosophie/*linguistique] que la vraie vie est celle de la vision et du rêve..., (Frantext). Le statut de recteur des SN cette croyance / philosophie peut s'expliquer par l'existence d'un sens permettant l'évaluation ou la caractérisation d'un état de choses. Avec ces SN, on peut caractériser l'état de choses exprimé par la subordonnée, en le qualifiant de croyance (l'adhésion de l'esprit à une opinion, une foi, etc.) ou de philosophique (conception ou mode de pensée que l'on se fait des problèmes ou de la vie), propriétés de caractérisation que n'ont pas les noms religion et linguistique. De par leur propriétés sémantiques, les noms croyance et philosophie permettent au locuteur d'exprimer son attitude ou son opinion vis-à-vis d'une proposition ou d'un état de choses quelconque. Ainsi, la subordonnée que la vraie vie est celle de la vision et du rêve est caractérisée comme étant la croyance de l'auteur italien ou du moins celle qu'il voulait véhiculer. Le SN cette philosophie peut être utilisé à sa place, dans la mésure où il caractérise aussi que $P$ comme étant une attitude, un mode de pensée qui guide le choix de l'auteur, d’autres noms comme idée, conception, etc. peuvent également être utilisés dans cet énoncé.

Nous considérons donc le nom recteur comme un élément porteur de modalité, cette même modalité se retrouvant dans l'interprétation du rôle discursif de la complétive (cf. Chevalier et Léard 1996 ; Ballier 2007 et Perkins 1983 pour l'anglais). La construction complétive est en effet une opération de caractérisation modale du contenu propositionnel de la part du locuteur. Cela s’observait déjà dans la définition que donnaient Wagner et Pinchon (1962 : 560) de la complétive nominale : « Pour le sens [1]e 
substantif [c'est-à-dire le nom recteur] marque que le contenu de la proposition conjonctive est posé comme un fait, ou bien qu'il est l'objet d'une énonciation, d'un jugement, d'un sentiment. » En interprétant ce passage, on peut estimer que non seulement le nom recteur a pour fonction de se référer au contenu propositionnel (le substantif marque le contenu), mais sert surtout à déterminer le statut conceptuel dudit contenu (il le présente comme un fait, comme l'objet d'une énonciation, d'un jugement, etc.). Nous estimons donc que le nom recteur entretient deux types de relations sémantico-pragmatiques avec la complétive qu'il régit: (1) la modalisation lexicale [+MODALITE], à savoir l'expression de l'opinion ou d'un jugement conceptuel du locuteur ou de l'énonciateur-origine par rapport au contenu de $P$, et (2) une référence " endophorique » [+ENDOPHORICITE], c'est-à-dire une relation de référence intralinguistique entre le SN et la subordonnée. Ce terme dérive du concept «d'endophore », lorsqu'un élément linguistique fait référence à un segment de discours, par opposition à " exophore » lorsqu'un élément linguistique renvoie à une entité située hors du discours ${ }^{11}$.

Le nom recteur de par ses propriétés de « marqueur modal » du contenu propositionnel permet au locuteur d'exprimer son attitude ou son opinion vis-à-vis d'une proposition, d'un événement ou d'un état quelconque. Par exemple, lorsque le locuteur dit (la philosophie que $P$ ), il procède à une caractérisation plus ou moins modale du contenu propositionnel. La proposition - que la nature est bonne, où les techniques de l'homme ne sont pas les bienvenues... - est présentée comme une philosophie qui guide l'attitude, le mode de pensée ou de croyance de certaines personnes dans la préservation de l'environnement.

Cependant, il faut noter que le trait [+MODALITE] ne suffit pas toujours pour qu'un nom régisse une complétive, certains noms peuvent avoir ce trait, mais ont en plus besoin d'un verbe support ou d'autres structures comme les présentatives (il y $a$, voici, etc.) pour régir la complétive. Chevalier et Léard (1996 : 55) indiquent par exemple que des noms comme besoin, souhait, vœu sont essentiellement dépendants de verbes supports. Cela montre que la contrainte de sélection relève aussi bien des propriétés sémantiques (modalité intrinsèque au nom) que des relations syntagmatiques.

Poser le problème sous cet angle soulève une autre interrogation : qu'en est-il de la valeur de modalité des modes et des autres éléments lexicaux ou grammaticaux ? Cette question est d'autant plus pertinente que de nombreux travaux ont souligné que le mode est un marqueur grammatical de la modalité. Il faut donc démontrer comment d'une part le type de modalité que marque le nom recteur dans la complétive, et d'autre part les rapports qu'il entretient avec les autres éléments marqueurs de modalité tels que le mode, les adjectifs, les auxiliaires ou verbes modaux. Sachant qu'en plus de la valeur modale du nom recteur, ces éléments apportent eux aussi la leur. Par exemple l'adjectif dans (la triste vérité est que) introduit une évaluation négative. Ce qui révèle par ailleurs que les modifications que subit le nom recteur ne sont pas uniquement d’ordre syntaxique, mais relèvent aussi des propriétés sémantiques et pragmatiques.

\subsection{La contrainte de modification}

Parmi les contraintes qui pèsent sur l'emploi des complétives, la modification est celle qui semble avoir reçu le moins d'attention. Et pourtant, elle est aussi présente et complexe que les autres puisqu'elle dépend non seulement du type de nom recteur, de son sémantisme et surtout de la nature de la relation qu'il entretient avec la complétive. En effet, l'analyse de quelques énoncés révèle que cette contrainte dépend en grande partie du type de relation prédicative entre le nom recteur et la complétive. Lorsque cette relation est complexe (élément support $+S N$ que), les possibilités de modification semblent plus grandes. Ce qui expliquerait par exemple la grammaticalité de l'adjectif folle avant le nom dans (21), mais aussi d'inquiétant et étrange dans l'énoncé (22b/c), mais pas dans (23b) qui relève d'une rection simple du type $S N+$ que $P$. Le doute sur le choix de l'adjectif fou (22b) ne relève pas de la relation prédicative, mais plutôt de la compatibilité entre le sémantisme du nom recteur et de l'adjectif lui-même.

(21) Madame de Rénal eut la folle idée que cet avertissement n'était qu'un prétexte pour ne pas la voir. (U. Virginia-Etext ${ }^{12}$ ),

(22a) Le fait est que ce matin la porte est bloquée. (Togeby 1985 : 98). 
(22b) Le fait inquiétant/étrange/??fou est que ce matin la porte est bloquée.

(22c) Les enfants me signalent le fait inquiétant/étrange/??fou que ce matin la porte est bloquée.

(23a) Le fait que ce matin la porte soit bloquée m'inquiète.

(23b) Le fait *inquiétant/*étrange/*fou que ce matin la porte soit bloquée m'inquiète.

Ainsi, le contraste entre les énoncés (22b/c) et (23b) indique-t-il que la contrainte de modification serait liée aux types de structures. On constate par exemple que le nom fait semble être plus modifiable par un adjectif lorsqu'il apparaît dans une relation de rection complexe (22b/c), notamment attributive et [V (support) + SN que], que quand il est dans une rection simple $[S N+q u e](23 \mathrm{~b})$.

D’autres noms semblent se soumettre à ces mêmes contraintes liées au type de structure lexicosyntaxique régissant la complétive. Tout comme dans les exemples précédents, la modification des noms vérité et idée par un adjectif est contrainte dans (24b et 26c) lorsque la complétive est régie par un nom sans un élément support [SN + que $P]$. Cette contrainte n’apparaît ni dans les exemples (24a et 26b), où il s'agit d'une structure attributive en $[S N+\hat{e} t r e+q u e P]$, ni dans (25 et 26a), où nous avons affaire à une structure en $[$ Vsup $+S N$ que $P]$.

(24a) La triste vérité est qu'il est fou. (Wilmet 1997 : 556)

(24b) *La triste vérité qu'il est fou...

(25) Madame de Rênal eut la folle idée que cet avertissement n'était qu'un prétexte pour ne pas la voir. (U. Virginia-Etext)

(26a) M. Hill, ambassadeur en Macédoine et médiateur entre les autorités de Belgrade et les responsables albanais du Kosovo, a transmis à $\mathrm{M}$. Milosevic «la très forte conviction de Mme Albright que l'offensive serbe en cours ne fait qu'accroître les risques d'une opération militaire de l'OTAN ». (U. Calgary-Le Monde 1998)

(26b) La très forte conviction de Mme Albright transmise à M. Milosevic est/était que l'offensive serbe en cours ne fait qu'accroître les risques d'une opération militaire de l'OTAN.

(26c) ??La très forte conviction de Mme Albright que l'offensive serbe en cours ne fait qu'accroître les risques d'une opération militaire de l'OTAN a été transmis à M. Milosevic.

Bien que la modification par un adverbe ne retienne pas prioritairement notre attention dans cette analyse, l'emploi de l'adverbe très dans l'énoncé (26a) mérite d’être souligné. Il constitue le prototype même de la double modification : un adjectif modifiant le nom recteur et est lui-même modifié par un adverbe. Une autre modification du nom recteur, assez courante, s'effectue par le biais d'une subordonnée relative comme dans (il y a une chose qui est fâcheuse dans votre cours, que tout le monde y prenne la liberté de parler, Wilmet 1997 : 557). Le nom recteur chose est modifié par la relative qui est fâcheuse dans votre cours. Nous ne traitons pas ici des éventuelles contraintes d'ordre entre relatives et complétives ${ }^{13}$, ni du statut déterminatif des relatives dans ce type de post-déterminations multiples.

Enfin, notons qu'outre la structure syntaxique, nous avons remarqué dans l'exemple (22b) que l'emploi d'un adjectif avec le nom recteur, comme dans toute modification adjectivale, relève aussi de propriétés sémantiques des deux éléments. A cet égard, la question de la contrainte de modification doit être traitée dans le cadre plus général de l’interface syntaxe / sémantique.

\section{Conclusion}

Cette étude montre la nécessité de distinguer les différents types de complétives afin de mieux saisir les contraintes en jeu dans ces constructions. Elle montre notamment que la contrainte de rection résulte d'un ensemble de relations sémantiques entre constituants que nous proposons de formuler comme un trait [+MODALiTE] permettant au nom de régir une complétive. Toutefois, elle n’est pas le seul élément en 
cause dans les restrictions de sélection du nom recteur. La structure lexico-syntaxique de la phrase joue également un rôle déterminant dans la sélection de certains noms recteurs. C'est dire que les contraintes liées à la construction de la complétive finie sont à la fois d'ordre modal et syntaxique.

Ainsi, la modalité et les propriétés distributionnelles des noms recteurs constituent un dénominateur commun aux restrictions de sélection et aux contraintes syntagmatiques. La structure de l'énoncé et la position syntaxique du nom recteur sont déterminantes dans la sélection d'un nom comme recteur. Par exemple, le passage d'une rection simple à une rection complexe peut permettre ou bloquer le choix d'un nom ou d'un adjectif dans un syntagme nominal. L'analyse suggère que la contrainte de modification par un adjectif dépend en particulier de la structure de la complétive nominale, même si, là encore, le sémantisme des éléments formels peut être en amont une source de contrainte. Les structures qui admettent la modification par adjectif sont les complétives à prédicats nominaux complexes (le cas de verbes supports, de présentatives, de syntagmes prépositionnels et de la structure attributive). L'étude propose donc une vue d'ensemble des problèmes que pose l'emploi des noms recteurs dans les différents types de complétives finies, en insistant sur la prise en compte des particularités qu'ont ces complétives et les propriétés distributionnelles des noms recteurs si l'on veut comprendre le fonctionnement global des complétives du nom. 


\section{Références bibliographiques}

Ahern, A. (2005). Mood Choice and the Sentence Interpretation in Spanish. Cahiers Chronos 13, pp.201-214.

Ballier, N. (2007). La Complétive du nom dans le discours des linguistes. In La Coordination et la subordination dans le texte de spécialité, sous la direction de David Banks. Paris: l’Harmattan, pp. 55-76.

Boone, A. (1994). La Complétive : un cas de nominalisation externe? TdL, 27, pp. 29-42.

Boone, A. (1996). Les Complétives et la modalisation. Linguistische Arbeiten 351, pp. 45-51.

Celle, A. (2006). Temps et modalité : l'anglais, le français et l'allemand en contraste. Berne : Peter Lang.

Chevalier, G. \& Léard, J. M. (1996). La Subordination nominale: classes, sous-classes et types sémantiques. Linguistische Arbeiten 351, pp. 53-65.

Grevisse, M. (1993). Le Bon Usage : Grammaire française. Goosse, A. (13eme éd.), Paris : Duculot.

Gross, G. (1996). Les expressions figées en français : noms composés et autres locutions. Paris : Ophrys.

Gross, M. (1975). Méthode en syntaxe : régime des constructions complétives. Paris : Hermann.

Herbst, Thomas; David, Heath; Ian F. Roe and Dieter Götz 2004. A Valency Dictionary of English. A Corpus-Based Analysis of the Complementation Patterns of English Verbs, Nouns and Adjectives. Berlin: Mouton de Gruyter.

Kanté, I. (2013).Typologie fonctionnelle des complétives nominales et analyse critique de quelques grammaires françaises et anglaises. Paris : Information Grammaticale n ${ }^{139}$, pp. 26-34

Kanté, I. (2011). La complétive nominale finie : entre syntaxe et sémantique. une étude contrastive anglaisfrançais. Thèse de doctorat à l’Université Paris 13, sous la direction du Professeur Nicolas Ballier.

Kanté, I. (2010). Mood and modality in finite noun complement clauses: A French-English contrastive study. In M. Stefania; K. Heylen and G. De Sutter (eds.), Corpus Studies in Contrastive Linguistics. IJCL 15: 2. Amsterdam: John Benjamins, 267-90.

Khalifa, J. Ch. (2004). Syntaxe de l'anglais - Théories et pratique de l'énoncé complexe au concours. Paris : Ophrys.

Korzen, H. (2003). Subjonctif, indicatif et assertion ou: Comment expliquer le mode dans les subordonnées complétives ? In M. Birkelund, G. Boysen and P.S. Kjærsgaard (eds.), Aspects de la modalité. Tübingen: Max Niemeyer Verlag, 113-129.

Kupferman, L. (1996). Observations sur le subjonctif dans les complétives. Linguistische Arbeiten 351, pp.141-151.

Lapaire, J.-R. et Rotgé, W. (1991). Linguistique et grammaire de l'anglais. Toulouse : Presses Universitaires du Mirail.

Le Goffic, P. (1993). La Grammaire de la phrase française. Paris : Hachette.

Leclère, C. (1971). Remarques sur les substantifs opérateurs. Langue française 11, pp.61-76.

Martin, R. (1983). Pour une logique du sens. Paris : PUF.

Muller, C. (1996). La Subordination en français : le schème corrélatif. Paris : Armand Colin.

Muller, C. (2008). Les bases de la syntaxe : syntaxe contrastive : français, langues voisines. Pessac: Presses universitaires de Bordeaux, $2^{\mathrm{ème}}$ édition.

Perkins, M. R. (1983). Modal Expressions in English. London : Frances Pinter Publishers.

Riegel, M. ; Pellat, J.-C. et Rioul, R. (1994). Grammaire méthodique du français. Paris : PUF.

Sandfeld, K. (1965). Syntaxe du francais contemporain. (2e éd.), Genève : Droz.

Soutet, O. (2000). Le Subjonctif en français. Paris : Ophrys.

Togeby, K. (1985). Grammaire française : la structure de la proposition. Volume V, Copenhague : Etudes Romanes de l’Université de Copenhague. 
Wagner, R. L. et Pinchon, J. (1962). Grammaire du français classique et moderne. Paris : Hachette.

Wilmet, M. (1997). Grammaire critique du français. Bruxelles : Duculot.

\begin{abstract}
* Nous tenons sincèrement à remercier les relecteurs anonymes du comité scientique du CMLF-2016 de leurs observations pertinentes et constructives. Nos remerciements vont également à Nicolas Ballier et à Véronique Bonnet pour leur lecture attentive et leurs observations sur les versions antérieures de cet article.

1. Ce qui n'est pas toujors le cas dans certains travaux, voir Riegel et al (1994), Leclère (1971), par exemple.

2. À noter que les complétives antéposées ne font pas l’objet d'une catégorie spécifique dans notre typologie, car il nous semble que des énoncés comme (1. Que l'humanité n'est pas belle, on le sait. Grevisse 1993: 1615 / 2. Que le vieil Horace est le personnage principal de cette tragédie, c'est la vérité. Grevisse 1993: 1615 / 3. Qu'il se trompe est certain. Grevisse 1993: 1594) sont, d'une manière ou d'une autre, des formes de dislocation à gauche de certaines des catégories décrites ci-dessus. Par exemple, en changeant la position des propositions de ces énoncés par extraposition ou structure attributive, on obtient respectivement une complétive du verbe, du nom et de l'adjectif (1'. On sait que l'humanité n'est pas belle. 2'. La vérité est que le vieil Horace est le personnage principal de cette tragédie. 3.' Il est certain qu'il se trompe.) Les énoncés ainsi obtenus peuvent être fonctionnellement différents de leurs équivalents antéposés en termes de focalisation, mais on a affaire à des formes qui correspondent à l'ordre $\mathrm{S}+\mathrm{V}+\mathrm{O}\left(1^{\prime}\right)$, attribut du sujet (2') et extraposée (3'). Dans (1 et 2), où la complétive est respectivement COD et attribut du sujet (ou sujet selon la structure), elle est reprise par les anaphorique « ce » et « le ».
\end{abstract}

3. (U. Calgary) concordance-corpus de University of Calgary: http://fis.ucalgary.ca/alle/fr.html

4. Voir Gross (1996) sur le verbe support et Kanté (2013 et 2011) pour une analyse plus détaillée des complétives impliquant ce type de verbe et leur rapport au SN recteur.

5. Mary-Annick Morel (communication personnelle) nous a signalé que l'appellation «complétive nominale " ne serait pas adaptée pour la structure extraposée, dont la subordonnée joue plutôt le rôle de sujet. Néanmoins, nous retenons cette structure dans la typologie des complétives pour montrer que, comme dans les vraies complétives, l'intégration syntaxique de la subordonnée en que se fait principalement via le nom recteur. En outre, les noms apparaissant dans la structure extraposée en que ont en commun avec les noms recteurs de complétives les mêmes contraintes sémantiques de sélection.

6. Voir Gross (1996) et Kanté (2011) pour une analyse détaillée de cette problématique.

7. Voir Leclère (1971) et Gross (1975).

8. Le nom affirmation, lorsque la proposition introductive n'est pas à la forme négative, et bruit dans Paul colporte le bruit que Marie viendra/*vienne. Voir Gross (1975: 53-54)

9. Celle (2006 : 91).

10. Les noms philosophie et linguistique peuvent référer à des disciplines, mais, pendant que linguistique a globalement cette seule référence principale, le terme philosophie, en plus de sa référence à la discipline, peut aussi signifier «manière de voir, de comprendre, d'interpréter le monde, les choses de la vie, qui guide le comportement / conception de quelque chose qui repose sur un ensemble de principes.... C’est précisément ce sens qui permet à ce dernier de régir une complétive. (voir Le Larousse de la langue française)

11. Nous empruntons ces notions à Lapaire et Rotgé (1991 : 53-54), à la suite de Halliday et Hasan (1976). Pour plus de détails sur cette analyse, voir Kanté (2011).

12. Le rouge et le noir, repris dans le corpus en ligne, http://etext.lib.virginia.edu/collections/languages/french/

13. Voir Ballier (2007). 